\title{
Image Processing Based Computational Tools for Assisting and Assessing Memorization and Learning Tasks
}

\author{
Mohammad Tanvir Parvez, Sameh Otri \\ Computer Engineering Department, Qassim University, Qassim 51477, Saudi Arabia.
}

Received: 22 April 2017; Accepted: 13 May 2017; Published: 08 November 2017

\begin{abstract}
In this paper, we present a novel computational framework for assisting and assessing memorization tasks. Such a framework can be used in any cases where certain level of memorization is needed, like in memorizing words/sentences, learning (programming) language structures, etc. We aim to identify the common memorization steps followed in various disciplines and then automate some of these steps to enhance memorization process. Particularly, we focus on annotation of texts (used for memorization) based on state of the art image processing techniques. Once texts are annotated and optionally commented, personalized tests can be automatically generated, focusing on the weakness of a particular student. These tests can further enhance the memorization process. As a case study, we have implemented the framework for a classical example of memorization: memorizing the Qur'an, the sacred book in Islam. Qur'an memorization is a well-known process since the early days of Islam and represents an ideal case for implementing the proposed framework.
\end{abstract}

Index Terms: Memorization tools, image processing, computational learning framework.

(C) 2017 Published by MECS Publisher. Selection and/or peer review under responsibility of the Research Association of Modern Education and Computer Science.

\section{Introduction}

Memorization is a critical concept of learning and important for any educational goals [13]. As a learning style, memorization through much repetition and guided practice yields to considerable achievements. However, there is some recent emphasis on the importance of understanding word meanings before memorizing [6, 16]. Viewing memorization as an effective learning style, there is a significant relationship between students' achievements in mathematics and science courses and 'memorization' [8]. According to some researchers, memorization could empower the human mind and enhance human knowledge [17].

In this paper, we aim at designing and developing a computational framework for teachers/learners for the

* Corresponding author.

E-mail address: m.parvez@qu.edu.sa, s.otri@qu.edu.sa 
purpose of enabling and improving their memorization tasks. Adopting this framework may enable students to be intellectual, immutable, and fast learners. The novel aspect of this framework is the use of state of the art image processing techniques for annotation of text and then generation of personalized tests. In this respect, learners and teachers need to be selective, by associating understanding and critical thinking with this memorization framework.

A number of learning theories are incorporated while formulating the proposed framework [5, 16]. These include: Game-based learning (interactive learning with a goal to retain what was learnt), Rote learning (a memorization technique based on repetition), Chunking approach (making more efficient use of short-term memory by grouping information), amongst others.

We have utilized our experience as teachers and learners for decades to formalize the memorization process in a framework that can be computationally implemented. As a case study, we have implemented the proposed framework for a classical example of memorization: memorizing the sacred text of Islam, the Qur'an. Qur'an memorization is a central activity in the lives of Muslim all over the world for over 1400 years. Many Muslims in the world aim to memorize the entire Qur'an by heart. Although, young children tend to memorize Qur'an faster than older people, many adults do also memorize the whole Qur'an at a later stage in their lives. Over these long periods of learning and memorizing Qur'an, certain 'standard' procedures have evolved to facilitate the learning process. These procedures, as will be discussed later, are not far from procedures followed in many other non-religious disciplines.

Therefore, the proposed framework is a result of our observations (as teachers/learners) in various Qur'an classes and the interviewing of several specialized Qur'an teachers. These observations gave us a first-hand experience of how students memorize and what techniques do work for them. In addition, we have utilized several learning theories from the literature $[1,16,18]$ while building the proposed framework. With the advancement in technology and mobile applications, Qur'an memorization has been facilitated by a number of multimedia interactive software [16]. However, these multimedia software do not take into account the annotation process and the need for personalized tests into their design.

Therefore, the contributions of the present work can be summarized as follows:

- Computational model for memorization process based on image processing techniques enabling interactive annotation, linking and tagging of texts.

- Automatic generation of personalized tests based on annotations, alleviating the immediate need for an examiner.

- Implementation of the framework for a classical example of memorization.

The rest of the paper is organized as follows. In Section 2, we review some learning theories, emphasizing the role of memorization in learning. The proposed computational framework is discussed in Section 3. Section 4 presents our implementation of the framework. Finally, Section 5 is the conclusions.

\section{Related Works}

In this section, we review some works on learning styles and methods. The goal here is to formalize the memorization process in general, so as to arrive at a framework that can be computationally implemented.

Leading theorists defined 'learning style' as the composite of characteristic style dimensions; cognitive, affective, and physiological that serve as relatively stable indicators of how a learner perceives, interacts with, and responds to the learning environment [15]. Learning styles depend on three factors; the leaner himself, the nature of subject or material to learn, and the environment and culture where the materials are taught.

The Learner: while learning in early years should give strong emphasis to memory, imitation, and repetitive practice, students in middle and advanced age should establish a connection and understanding to the material being learnt [1]. In their early years, Chinese children are taught to learn through memory, imitation and repetitive practice [3]. 
Material / skills to be learnt: memorizing grammatical examples, extensive lists of English words, the whole text or even recite the whole of a good sized dictionary by heart are considered to be a passive learning. For example, even though many characters in Chinese language have clues to pronunciation and meaning in their component parts, the task of learning to read Chinese is mostly a matter of memory after teaching children how to use these clues [14].

Teacher / Environment / Culture: being an active student differs from one culture of learning to another and it should be taken into account. For example, in Middle East and China, active students should listen carefully to the teacher, take notes then review them later and memorize the vocabularies and grammar. However, in Europe, it means being active in talking and conversations. Some learners have to summarize the whole lesson first then memorize the summary. Language as communication is neglected and the excessive focus on teacher centred activities puts students into a passive role of listening and memorizing.

Thus, for the materials to be learnt, memorization process plays a pivotal role in many disciplines. This is true not only for very young learners (children learning a new language or memorizing new words), but also for more advanced learners. For example, programming languages are normally taught at a university or high school levels. Learning programming languages generally necessitates memorizing certain structures and rules. Memorizing such information needs repetition, practice and periodic test.

The following fifteen steps summaries a framework of memorization, as illustrated in Fig.1.

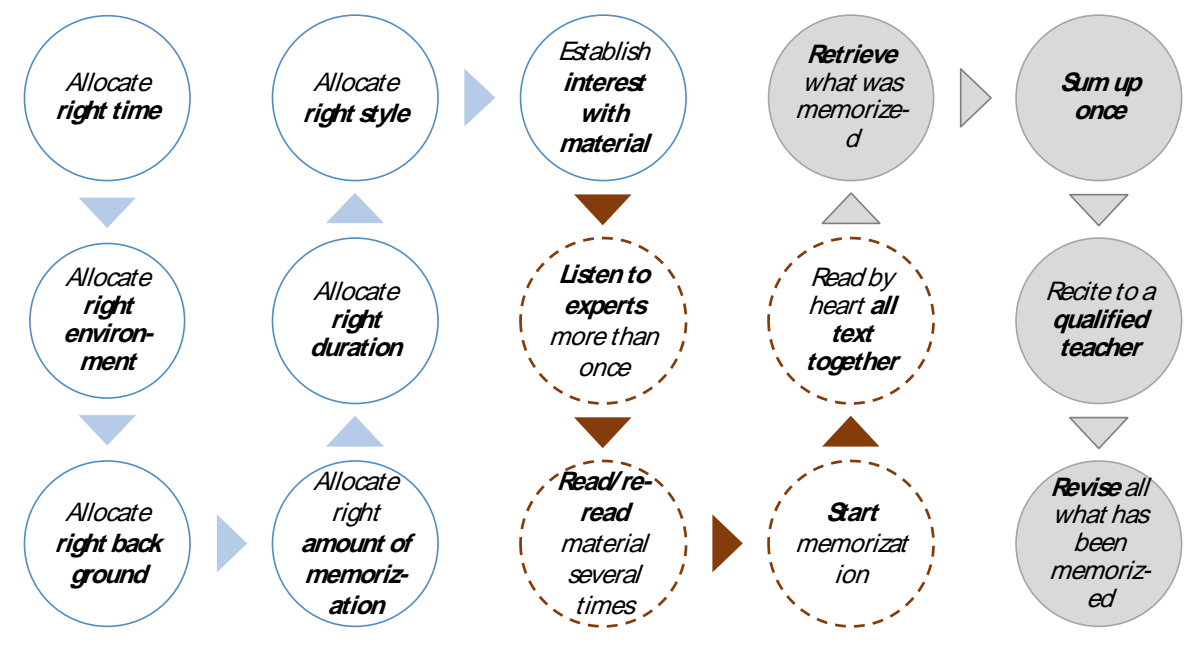

Fig.1. Illustration of Steps in Memorization of Texts.

This general framework comprises three main stages for the purpose of structured memorization.

\section{A. Pre-memorization:}

The first seven steps (shown as solid circles in Fig.1) includes choosing the right time, environment, amount of memorization and establish an interest of the memorized materials.

- Step 1: to select a suitable state of mind where mind is fresh and is able to memorize. This could be in the early morning after one slept very well with empty stomach. Or it may be just before one goes to the bed.

- Step 2: to select the right environment to sit and read, which could be a quiet or a noisy one.

- Step 3: to select the right background with music, no music or a melodic voice. 
- Step 4: to choose the right amount of pages according to the capacity of one's memory.

- Step 5: allocate the right duration of memorization which could an hour or so.

- Step 6: to choose the right style of reading such as normal conversation, reading aloud or quite.

- Step 7: to read the text more than once and read the meaning of it which helps to increase familiarity with the text.

\section{B. Memorization:}

The next four steps (shown as dotted circles in Fig.1) involves reading the text many times, trying to memorize it bit by bit and repeating it together.

- Step 8: it will increase the learning's listening skill and make sure that his/her recitation is accurate.

- Step 9: this will help keep a strong image of this text in the learner memory.

- Step 10: the learner starts with part of the text (if the material is Qur'an, then takes an Ayah), memorizes it, then takes the next part to memorize it; then he/she repeats both parts together and so on with rest of the parts.

- Step 11: read by heart all sentences/verses together until one feels comfortable with what one has memorized (with determination and persistence).

\section{Post-memorization:}

The last four steps (shown as filed circles in Fig.1) work as a confirmation of mastering what have been memorized.

- Step 12: retrieve what had been completely memorized in the next morning.

- Step 13: sum up once, what one had memorized for the whole week (as an example).

- Step 14: recite to a qualified teacher/ an expert listener/ a friend who can arbitrate/ judge one's memorization and reading at the same time (this can be a daily or weekly activity).

- Step 15: before starting a new phase of memorization, a learner should revise what has been memorized a day before (or last week in total) then join it with the new part after memorizing it.

This is a general framework which works for any learner and for any course. However, in some exceptions, some individuals may memorize any courses, and whether or not with further understanding nor for much preparation before memorizing. In addition, learners have to distinguish which needs to be memorized from that which does not.

With the advancement of information technology, many classroom settings now incorporate computational tools. For example, visualization tools have been used by several researchers both for teaching at advanced level [11] or for elementary levels [12]. However, these works mainly focus on building aides for learning, rather than capturing the whole process of learning and memorization.

More importantly, most of the automated memorization tools do not handle the post-memorization tasks, which heavily depend on tests and the availability of a teacher/judge. The proposed framework exactly focuses on this area: allow students to annotate what they are memorizing, with comments and tags on their various levels of difficulties on the subject matter, and then generate personalized tests for the students to test their learning. With the availability of image processing techniques, we have been able to build and implement such a computational framework, as discussed in the following sections.

In this work, we thus aim at automating the process of memorization and learning by utilizing image processing techniques with two goals in mind:

Goal 1: Enable students to annotate on what is being learnt or memorized, by linking and tagging. 
Goal 2: Generate personalized and adaptive tests on the material being learnt, alleviating the immediate need to a teacher/judge.

Based on these discussions, we describe the following computational framework to facilitate the memorization and learning process.

\section{Proposed Framework}

In this section, we discuss in details the proposed computational framework for memorization and learning. The major functionalities of the framework are shown in Fig.2. The whole process is divided into two main units: an image level pre-processing unit and a memorization unit that comprises of repetition, annotation and test. Both units are later elaborated in Fig. 3 and Fig.5. Both of these units heavily depend on image processing methods to accomplish their functionalities. The details of these two units are given below.

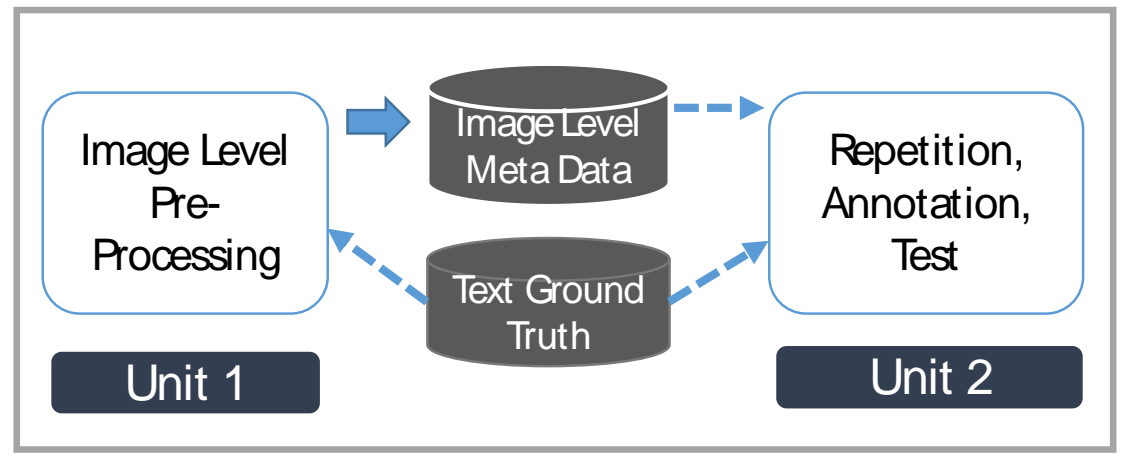

Fig.2. The Two Units in the Proposed Framework.

\subsection{Extraction of Image Meta Data}

As illustrated in Fig.3, the pre-processing steps in unit 1 focus on extracting some image level meta data. These data, in conjunction with ground truth (GT), are used in annotation and tagging processes in unit 2 . In this section, we explain how these meta data can be extracted from texts.

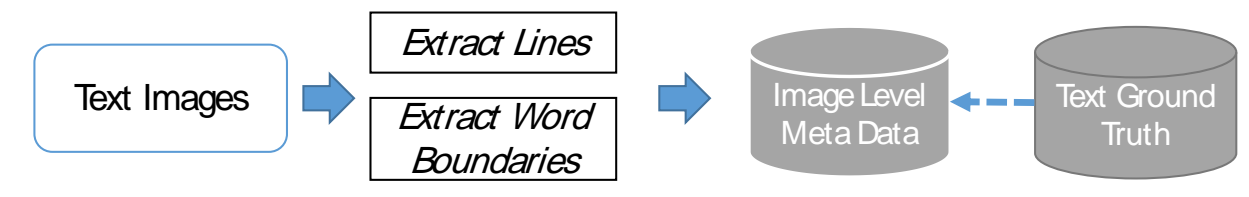

Pre-processing

Fig.3. Unit 1 of the Proposed Framework: Pre-Processing Steps before the Memorization and Learning Process Begins.

Assume that, the text to be learned/memorized can be represented by an image $I$. The image $I$ consists of $L$ lines of words. It is assumed that the goal of memorization is to memorize one or more lines in $I$, while the test can be carried out at the word or line level. In the pre-processing phase, we extract these $L$ lines from $I$ and also locate the word boundaries in each of the extracted lines. These word boundaries are then aligned with the GT. The following procedure summaries the process of extracting line level meta data from $I$. 


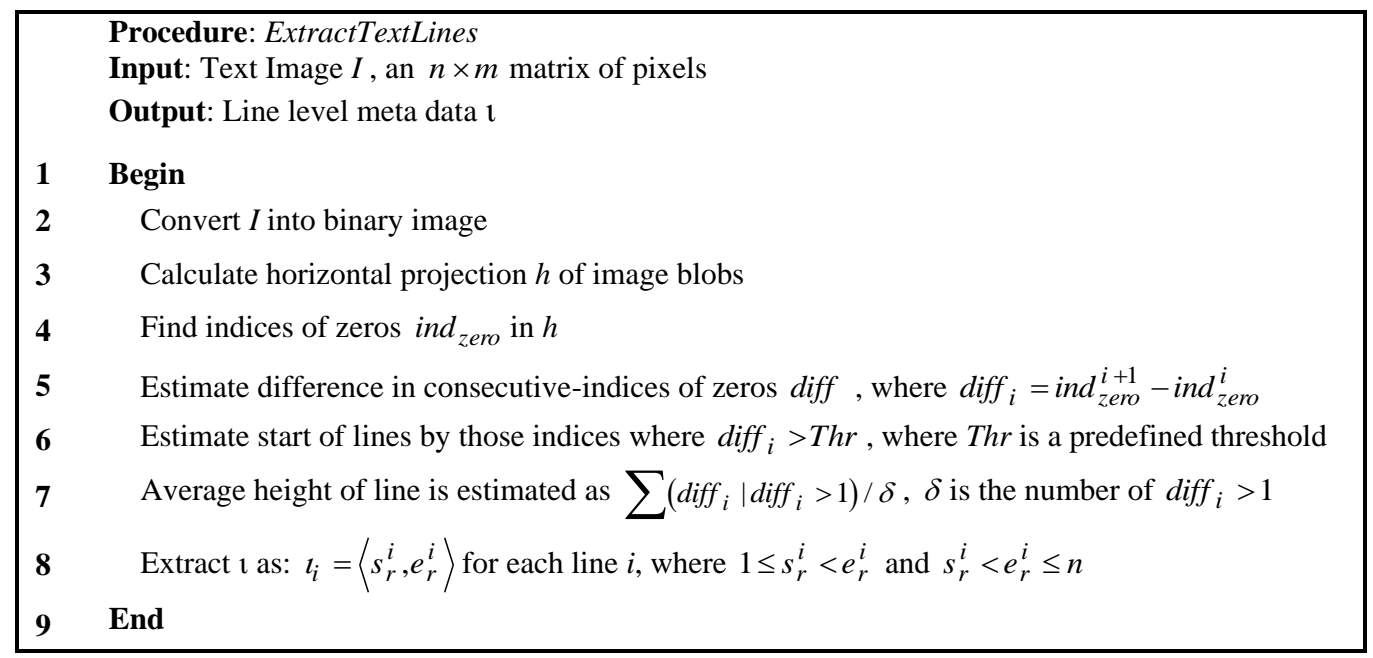

An example of running the ExtractTextLines procedure is shown in Fig.4. In Fig.4 (a), a sample code from $C$ programming language is shown as an image of text. This image of text is segmented to extract lines using horizontal projection as shown in Fig.4(b). Note that, in Fig.4(b), the empty spaces between lines result in gaps in the projection profile.

Procedure ExtractTextLines is more suitable for images of printed text. For text images containing handwritten text, a more complicated algorithm involving image-blobs processing and segmentation is needed. For more details on such algorithms, readers are referred to [10]. In our work, we have utilized the method discussed in [9].

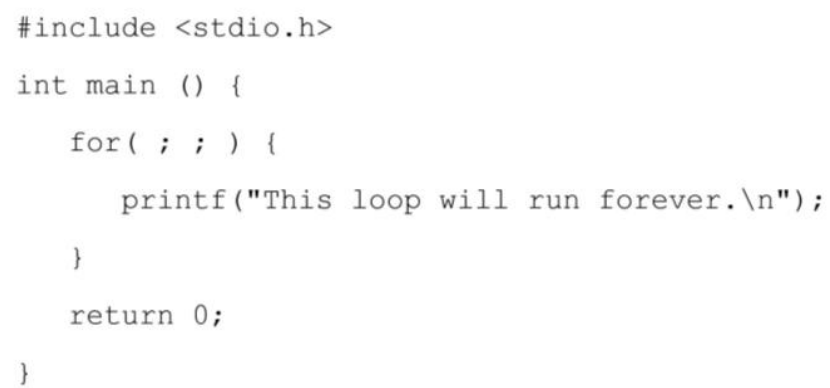

Fig.4. (a) A page of Code Explaining 'Loop' in $C$ and (b) horizontal Projections for Extraction of Lines.

Once the text lines are extracted from $I$, we identify the word boundaries in each line of $I$. The following procedure captures the process of identifying the word boundaries. The goal of LocateWordBoundaries procedure is to identify the bounding box for each word in image $I$. Each extracted word level meta data is then mapped to the ground truth (GT) data. GT contains the digital version of the text image $I$ and is used to generate the personalized tests from annotation and tagging, as discussed in the next section. 


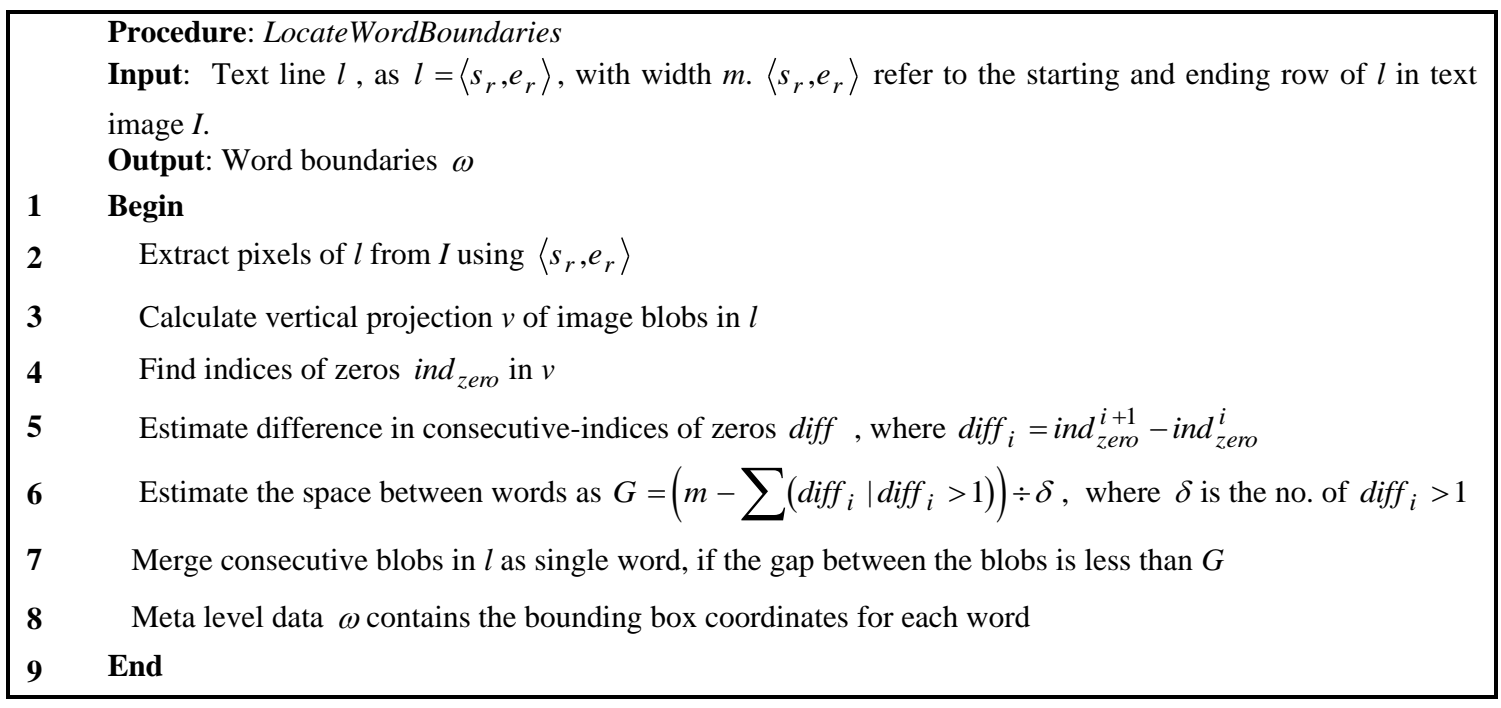

\subsection{Memorization Unit}

Unit 2 (shown in Fig.5) of the proposed framework comprises of three modules. In module 1, functionalities regarding listening and reading of text and tracking of progress are provided. Module 2 focuses on annotation and tagging of text at the image level. These annotations are mapped to image level meta data and saved in annotation database. Once annotations and tagging are completed, personalized test banks can be generated based on an annotation database and GT (module 3).

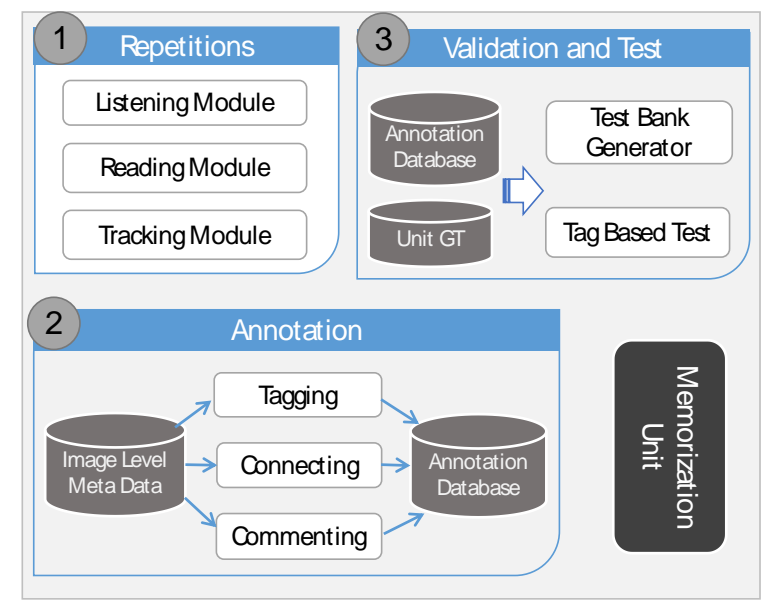

Fig.5. Unit 2 of the Proposed Framework: Three Main Modules of Activities in the Memorization Process.

The process of annotation is further illustrated in Fig.6. The learner is allowed to interact with the text at the image level. Once the learner decides to annotate any part of the text, he/she marks the rectangular area in the text image where annotation should be made. A marked rectangular area is tuple of the form rec $=\langle x, y, w, h\rangle$, where $\langle x, y\rangle$ is the top-left co-ordinate of the marked rectangle in image $I$. The width and height of the marked 
rectangle is given by $\langle w, h\rangle$. From the extracted meta data $\omega$ of text image, the marked rectangle is aligned/matched with the closest word(s) boundary. Once the proper annotation area is selected, tagging and commenting can be performed. Tagging options depend on the type of learning materials and can be listed while implementing the framework.

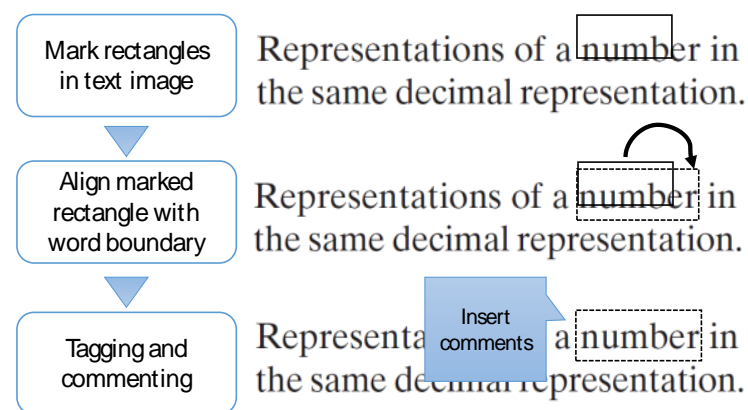

Fig.6. Illustration of the Process of Annotation and Tagging at the Text Image Level.

Tagging a selected area of text is a process of associating a set of Boolean statements to the selection. Assume that $\mathrm{B}$ is a set of statements, where each statement $\beta \in \mathrm{B}$ can be either True of False. Then the process of tagging is defined as selecting a Boolean string $\langle\dagger\rangle$, which a sequence of 0 s and $1 \mathrm{~s}$, where $|\langle\dagger\rangle|=|\mathrm{B}|$. The process of annotation and tagging is summarized in the following AnnotateTagTextImage procedure.

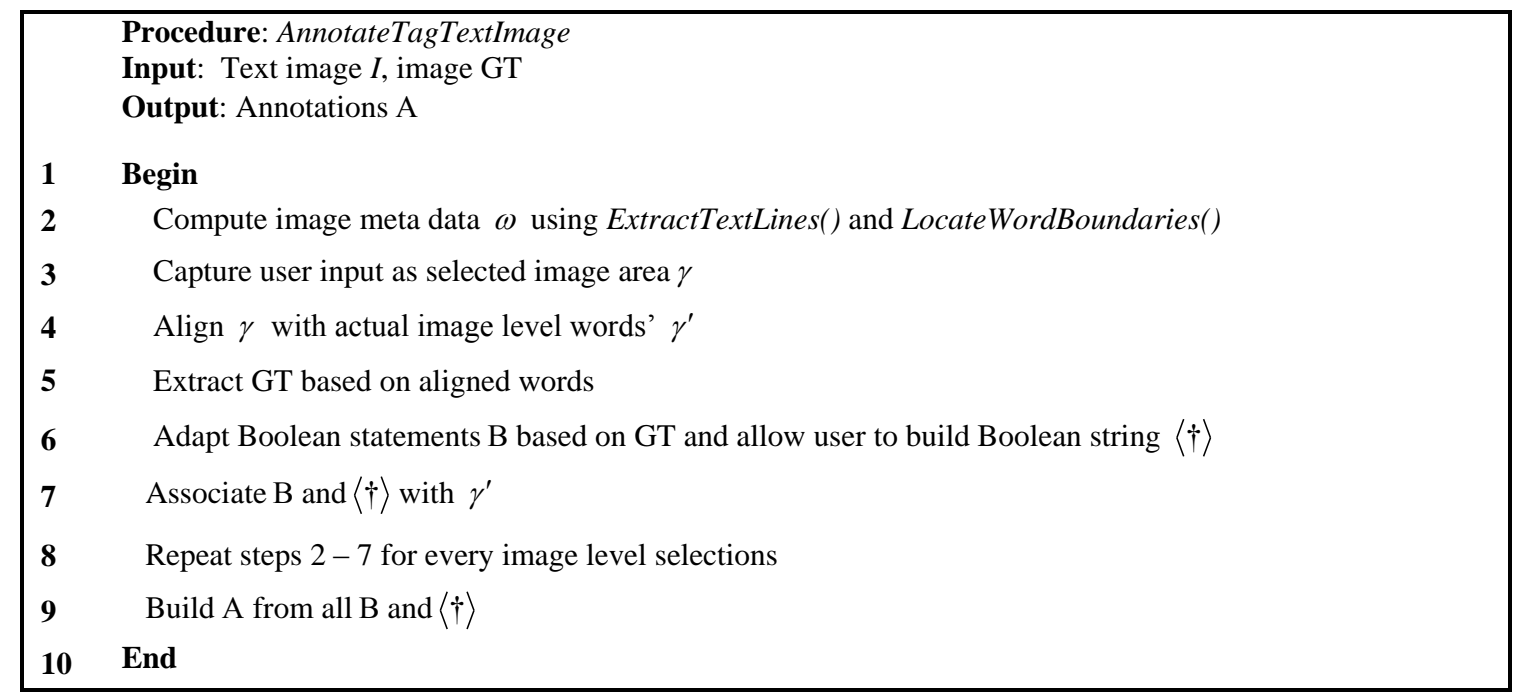

As an example for tagging, consider the case where a student marks words that he/she is memorizing. Suppose, once a word is marked, the student is presented with $\mathrm{B}=\{$ 'Iforget theword', 'Imakemistakeinspelling' $\}$. Now the student records his/her responses to these two Boolean statements and builds the string $\langle\dagger\rangle$, say as $\langle\dagger\rangle=\langle 0,1\rangle$. This response from the student means that he/she can remember the word, however he/she may make mistake in the spelling. These responses are stored in the annotation database and then can be used for generating test sets. Additionally, the student can record free-form comments. 
The purpose of tagging and commenting the selected text is manifold. Tagging can be used for categorization of errors, creating question banks, enabling self-assessments, lesson plan generation based on students' performance, etc. This is the purpose of module 3 in Fig.5. For the example mentioned above, a test can be generated for a student from the words marked as 'difficult to spell'. In addition, in a large set of text documents, the pattern of difficulties for a particular learner can be discovered. Interactive manipulation of texts can allow learners to connect different sections based on similarities or other relations.

In a multiuser environment, annotated and tagged data represent an ideal case for data analytics. In a class room setting, instructors can generate a generalized test that focuses on more common mistakes made by the learners.

The framework described in this section is implemented for a classical example of memorization task, as discussed next.

\section{Implementations}

In this section, we present our implementation of the proposed framework. We have implemented our framework for the memorization tasks of Qur'an, the sacred text in Islam. Qur'an is being memorized by Muslim all over the world since over a thousand of years; thus memorization of Qur'an presents an ideal case for our purpose.

For implementation purpose, we have taken images of Qur'anic text from Madinah Quran Printing Complex [7]. The ground truth values (the text of Qur'an) are taken from the highly authenticated version archived in [4]. Due to its wide support for image processing techniques, the implementation of the framework was programmed in MATLAB. The main interface of the developed system is shown in Fig.7 and is divided into a number of modules: listening and repetition module, progress tracking module and annotation module. These modules have been developed based on the discussions in the previous section. The listening and repetition module has functions required by (almost) all students of Qur'an and are selected based on the authors' experience in this field (along with the suggestions from experts).

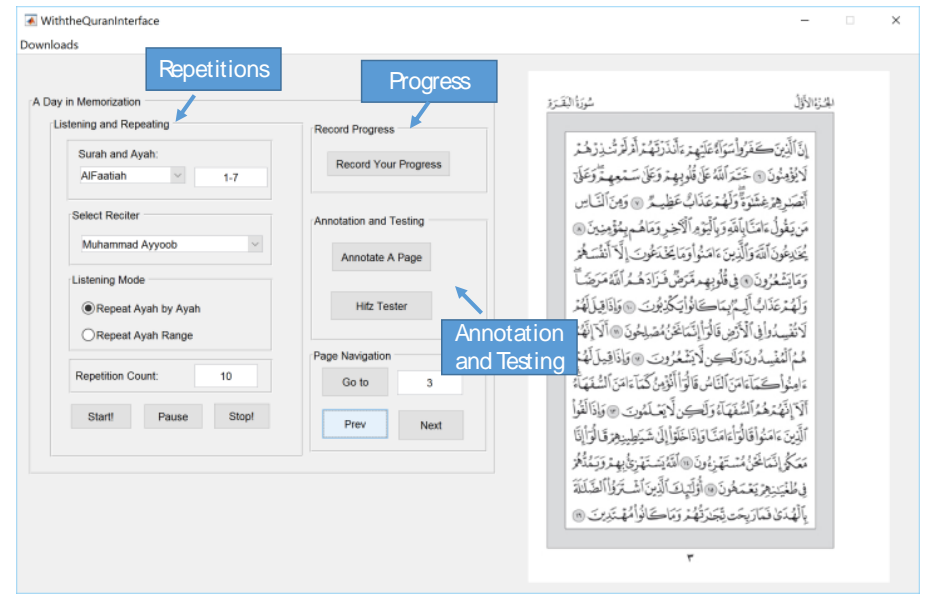

Fig.7. Illustration of the Main Interface for Memorization of Qur'an.

For each page of Qur'an, we first extract image level meta data using the procedures discussed earlier. These meta data include locations of ayah (sentence in Qur'an) boundaries, boundaries for each word in each ayah with their ground truth mapping, locations and numbers of ayah markers, etc. Once these meta data are extracted, users are allowed to interact with a page of Qur'an and annotate one or more words. The interface for annotation and tagging is shown in Fig.8, with the main functionalities marked. 


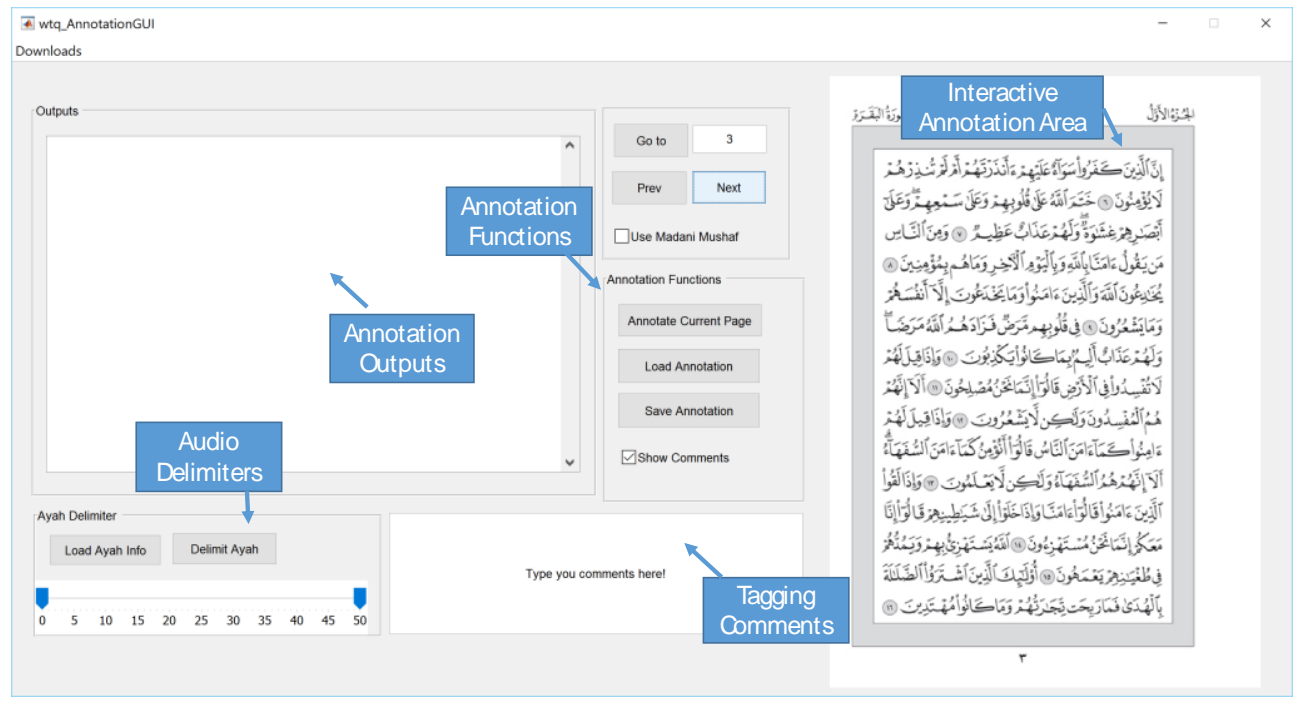

Fig.8. Interface for annotation, Tagging and Commenting.

Users are allowed to make new annotations, or load and edit old ones. The process of annotation starts with the user selecting portions (in rectangular shape) on the screen showing a page from Qur'an. The selected area on the page is then automatically mapped to the closest word(s) in the page. Then the user is given a number of choices regarding the selected text (that corresponds to the Boolean statements B ). An example of such options (used for tagging the selected text) is illustrated in Fig.9. Fig.10 illustrates the visual representation of an annotated page, once the user either completes the annotation process or loads any previously created annotations.

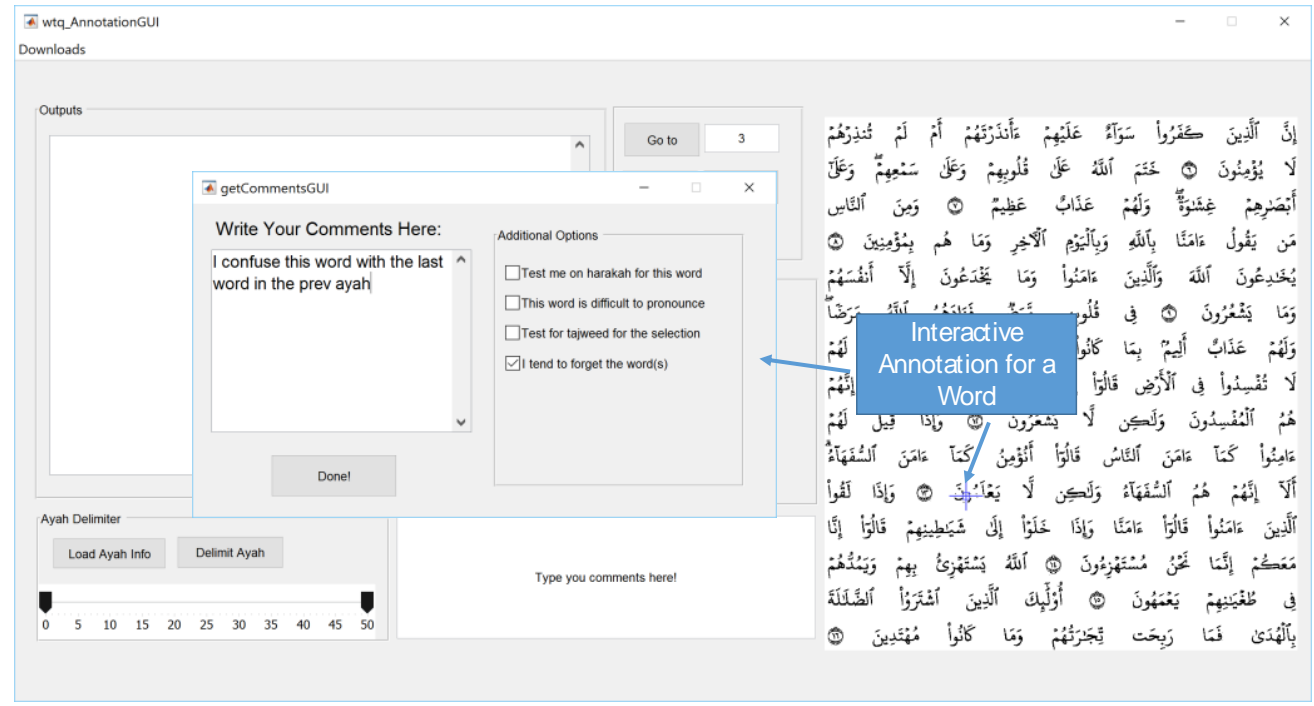

Fig.9. Interactive Selection of Text and Tagging and Commenting. 


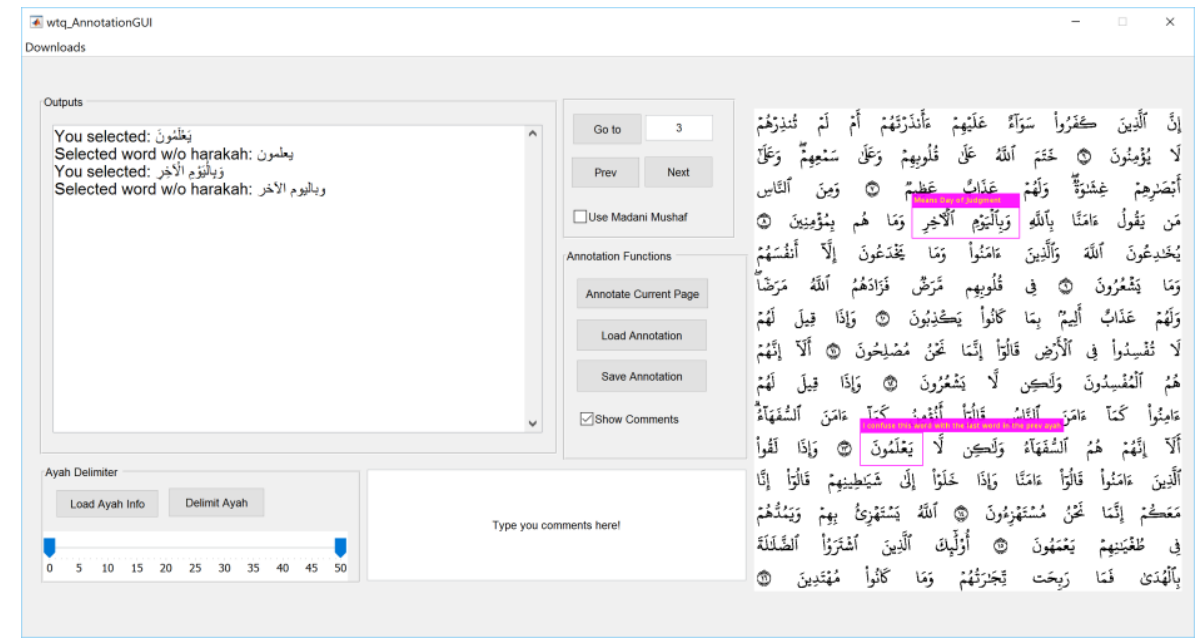

Fig.10. An Example of Annotated Text in Memorization of Qur'an.

Once annotations are created for one or more pages of Qur'an, personalized tests can be generated. One such example is given in Fig.11 where the system automatically tests the user on the words he/she tends to forget, which is one of the common challenges faced by the students in memorization of Qur'an.

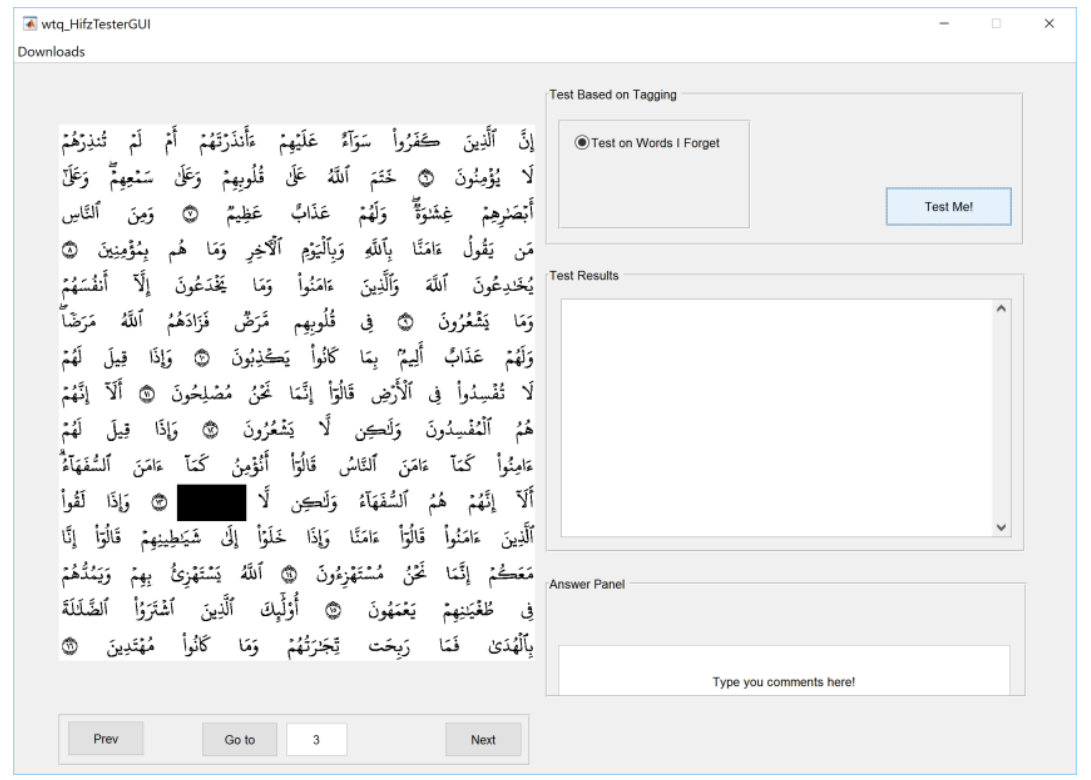

Fig.11. Generation of Personalized Tests Based on Tagging.

\section{Conclusions}

In this paper, we have presented a framework that can be used to build a computational tool to assist memorization of texts. The tool heavily depends on state of the art image processing techniques. We have implemented the framework for a classic case of memorization: memorizing the Qur'an. 
The implementation of the framework for a particular domain may need consultation from experts in that domain to identify the tagging options. For our implementation, we have utilized our own knowledge and some suggestions from the experts in Qur'an memorization.

Such a framework can be easily developed for tablets and smart phones for easier interactions.

\section{Acknowledgements}

This work was supported by the Deanship of Scientific Research, project no. 3296, Qassim University, Saudi Arabia.

\section{References}

[1] Biggs, J. (1996). Western misperceptions of the Confucian-heritage learning culture. In D. Watkins, \& J. Biggs (Eds.), The Chinese learner: Cultural, psychological, and contextual influences, Hong Kong: CERC \& ACER, pp. 45-67.

[2] Boyle, H. N. (2006). Memorization and learning in Islamic schools. Comparative Education Review, 50(3), pp. 478-495.

[3] Cortazzi, M; Jin, L. (1996) Cultures of Learning: Language Classrooms in China in H. Coleman (ed.) Society and the Language Classroom, Cambridge: CUP, pp.169-206.

[4] Download Quran Text, http://tanzil.net/download (accessed 12/7/2016).

[5] Elobaid M, Hameed K, Yahia Eldow ME. (2014) Toward designing and modeling of Quran learning applications for android devices. Life Sci J. 11(1), pp. 160-171.

[6] Little, J. L., \& McDaniel, M. A. (2015). Individual differences in category learning: Memorization versus rule abstraction. Memory \& cognition, 43(2), 283-297.

[7] King Fahd Glorious Quran Printing Complex, http://www.qurancomplex.org/ (accessed 12/5/2016).

[8] Park, C. C. (2000). Learning style preferences of Southeast Asian students. Urban Education.35(3), 245268.

[9] Parvez, M. T., \& Mahmoud, S. A. (2013). Arabic handwriting recognition using structural and syntactic pattern attributes. Pattern Recognition, 46(1), 141-154.

[10] Parvez, M. T., \& Mahmoud, S. A. (2013). Offline Arabic handwritten text recognition: a survey. ACM Computing Surveys (CSUR), 45(2), 23.

[11] Petersson, H., Sinkvist, D., Wang, C., \& Smedby, Ö. (2009). Web - based interactive 3D visualization as a tool for improved anatomy learning. Anatomical sciences education, 2(2), 61-68.

[12] Raiyn, Jamal. (2016). Developing a Mathematics Lesson Plan based on Visual Learning Technology, I.J. Education and Management Engineering, 4, pp. 1-9.

[13] Rashidi, N., \& Omid, A. (2011). A survey on Iranian EFL learners' beliefs on the role of rote memorization in learning vocabulary and its effect on vocabulary achievement. Journal of Pan-Pacific Association of Applied Linguistics, 15(1), pp. 139-161.

[14] Salili, F. (1996). Accepting personal responsibility for learning. In D. Watkins, \& J. Biggs (Eds.), The Chinese learner: Cultural, psychological, and contextual influences. Hong Kong: CERC \& ACER. pp. 85105.

[15] Smith, D. R. (2008). Learning style preference, sense of classroom community, gender, age, and previous experience within computer-mediated instruction (CMI). The University of North Carolina at Greensboro.

[16] Shamsuddin, S. N. W., Bakar, N. F. A., Makhtar, M., Isa, W. M. W., Rozaimee, A., \& Yusof, N. (2016). A Framework for Designing Mobile Quranic Memorization Tool Using Multimedia Interactive Learning Method for Children. Journal of Theoretical and Applied Information Technology, 92(1), 20.

[17] Soloso L, R. (1988). Cognitive Psychology. USA: Allyn and Bacon Inc. 
[18] Yusef, M., (2010), Memorization as a learning style: A balance approach to academic excellence, International Journal of Sustainable Development, 1(6), 49 -58.

\section{Authors' Profiles}

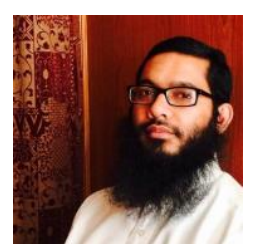

Mohammad Tanvir Parvez obtained his B.Sc. and M.Sc. Engineering in Computer Science and Engineering (CSE) from Bangladesh University of Engineering and Technology (BUET), Dhaka and a Ph.D. in CSE from King Fahd University of Petroleum \& Minerals (KFUPM), Dhahran, Saudi Arabia in 2010. His research interests include Pattern Recognition, Image Processing and Machine Learning with special interest in handwriting recognition using structural approach. He has received several awards including Best Graduate Students' Award (KFUPM, 2010), First Prize in Graduate Seminar Day (KFUPM 2010), BUET Academic Performance Scholarship, etc. Dr. Parvez is an Assistant Professor in Computer Engineering Department at Qassim University, Saudi Arabia since 2011.

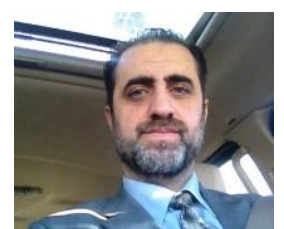

Sameh Otri obtained his B.Sc. in Electronic Engineering from Aleppo University in Syria and M.Sc. in Systems Engineering and $\mathrm{PhD}$ in Artificial Intelligence and Optimization Techniques from Cardiff University in the UK in 2010. His research interests include Robotics and Optimization techniques such as Bees Algorithm, Genetic Algorithms and Ant Colony Optimization. He has received several awards including 1st prize poster in Integr8or Doctoral Conference, Nottingham University, UK (2008), 1st prize poster in the Graduate School of Physical Sciences and Eng., Cardiff, UK () and $1^{\text {st }}$ Prize for best paper by a young researcher IPROMS Virtual Conference, UK (2006). Dr. Otri is an Assistant Professor in Computer Engineering Department at Qassim University, Saudi Arabia since 2013.

How to cite this paper: Mohammad Tanvir Parvez, Sameh Otri,"Image Processing Based Computational Tools for Assisting and Assessing Memorization and Learning Tasks", International Journal of Education and Management Engineering(IJEME), Vol.7, No.6, pp.1-13, 2017.DOI: 10.5815/ijeme.2017.06.01 